\title{
Matéria orgânica e aumento da capacidade de troca de cátions em solo com argila de atividade baixa sob plantio direto
}

\author{
Soil organic matter and cation exchange capacity increase in a low activity clay soil under no-tillage \\ system
}

\author{
Marlise Nara Ciotta ${ }^{1}$ Cimélio Bayer $^{2}$ Sandra Mara Vieira Fontoura ${ }^{3}$ Paulo Roberto Ernani $^{1}$ \\ Jackson Adriano Albuquerque ${ }^{1}$
}

\begin{abstract}
O sistema de manejo afeta a matéria orgânica do solo, o que pode ter expressivo efeito na CTC de solos com argila de atividade baixa. Neste estudo, avaliou-se o efeito da utilização durante 21 anos do sistema plantio direto (SPD) sobre os estoques de carbono orgânico (CO), bem como a sua relação com o aumento da CTC de um Latossolo bruno (629 $\mathrm{g} \mathrm{kg}^{-1}$ de argila), em Guarapuava, PR. O SPD promoveu acúmulo de CO na camada superficial do solo $(0-6 \mathrm{~cm})$, o que refletiu-se num aumento de 2,63t $\mathrm{ha}^{-1}$ no estoque de $C O$, na camada de $0-20 \mathrm{~cm}$, em comparação ao preparo convencional. A baixa taxa de acúmulo de $C O$ $\left(0,12 t\right.$ ha $^{-1}$ ano $\left.^{-1}\right)$ foi relacionada à alta estabilidade física da matéria orgânica neste solo argiloso e oxídico. Apesar do pequeno acúmulo de $C O$ no solo sob SPD, este teve reflexo positivo na CTC do solo, com um aumento médio, na camada de $0-8 \mathrm{~cm}$, de $15,2 \mathrm{mmol}_{\mathrm{cg}}^{-1}$ na CTC efetiva, e de 20,7mmolckg-1 na CTC a pH 7,0, em comparação ao solo em preparo convencional. Os resultados obtidos reforçam a importância do SPD quanto ao seu efeito nos estoques de matéria orgânica e, em consequência, na CTC de solos tropicais e subtropicais com predominância de argila de atividade baixa.
\end{abstract}

Palavras-chave: solos tropicais; atividade de argila; manejo de solo; qualidade do solo.

\section{ABSTRACT}

Soil management affects the organic matter stocks, and thus the CEC especially in low activity clay soils. The main goal of this study was to evaluate the long-term (21 years) effect of the no-tillage on soil organic carbon (SOC) stocks and its relationship with CEC increase in a clayey Oxisol
(Hapludox), in Guarapuava (PR), Southern Brazil. Notillage soil had only $2.63 \mathrm{t} \mathrm{ha} \mathrm{h}^{-1}$ more SOC than conventionally tilled soil at $0-20 \mathrm{~cm}$, and the highest net accumulation occurred in soil surface layers $(0-6 \mathrm{~cm})$. The low accumulation rate of SOC in the no-tilled soil $\left(0,12 t \mathrm{ha}^{-1} \mathrm{yr}^{-1}\right)$ was related to the high physical stability of soil organic matter in this clayey Oxisol. Despite the small effect on SOC contents, the no-tilllage had an expressive influence on the CEC of $0-8 \mathrm{~cm}$ soil layer. The effective CEC increased $15.2 \mathrm{mmolckg}^{-1}$ and the potential ( $\mathrm{pH}$ 7.0) CEC increased 20.7mmolckg-1 in this soil layer, in comparison to conventional tillage system. The results support the positive effect of no-tillage on soil organic matter stocks and on CEC of tropical and subtropical soils with predominantly low activity clay minerals.

Key words: tropical soils; clay activity; soil management; soil quality.

Em solos tropicais e subtropicais, a matéria orgânica apresenta uma estreita relação com as demais propriedades físicas, químicas e biológicas do solo. Portanto, o manejo sustentável da matéria orgânica do solo é fundamental à manutenção da capacidade produtiva do solo em longo prazo. O efeito do manejo sobre os estoques de matéria orgânica é dependente do tipo de solo. BAYER (1996) verificou uma diminuição praticamente pela metade da taxa de decomposição da matéria orgânica no solo em sistema plantio direto (SPD) em comparação ao sistema de preparo convencional (SPC), com reflexos positivos no aumento dos estoques de carbono orgânico (CO)

${ }^{1}$ UDESC, Departamento de Solos, CP 281, 88520-000, Lages, SC. Bolsista do CNPq.

${ }^{2}$ UFRGS, Departamento de Solos, CP 776, 9001-970, Porto Alegre, RS. Bolsista do CNPq. Autor para correspondência.

${ }^{3}$ Pesquisadora da FAPA-Fundação Agrária de Pesquisa Agropecuária, 85108-000, Guarapuava, PR. 
de um Argissolo Vermelho, com $220 \mathrm{~g} \mathrm{~kg}^{-1}$ de argila e mineralogia caulinítica. Entretanto, num Latossolo vermelho, com $680 \mathrm{~g} \mathrm{~kg}^{-1}$ de argila e mineralogia oxídica, o SPD diminuiu a taxa de decomposição da matéria orgânica em apenas 14\%, com pequeno reflexo nos estoques de $\mathrm{CO}$ do solo.

Apesar de menos pronunciado, o aumento nos estoques de matéria orgânica em Latossolos argilosos em SPD é suficiente para ocasionar aumento expressivo na capacidade de troca de cátions destes solos com predominância de argila de atividade baixa. Para testar esta hipótese, amostrou-se um Latossolo bruno (629 $\mathrm{g} \mathrm{kg}^{-1}$ de argila), em SPD e SPC, num experimento instalado em 1978, na área experimental da FAPA-Fundação Agrária de Pesquisa Agropecuária, em Guarapuava, PR. Duas sub-amostras de solo foram coletadas, em setembro de 1999, nas três repetições experimentais de cada sistema de manejo de solo, com auxílio de espátula, nas seguintes profundidades: 0-2, $2-4,4-6,6-8,8-10,10-15$ e $15-20 \mathrm{~cm}$. As amostras compostas de solo foram secas à sombra, moídas, peneiradas a $2 \mathrm{~mm}$, e analisadas em relação aos teores de $\mathrm{CO}$, teores de $\mathrm{Ca}, \mathrm{Mg}, \mathrm{K}$ e $\mathrm{Al}$ trocáveis (TEDESCO et al., 1995), e acidez titulável (H+Al) (EMBRAPA, 1997). A CTC efetiva foi calculada pela soma dos teores de $\mathrm{Ca}, \mathrm{Mg}, \mathrm{K}$ e $\mathrm{Al}$ trocáveis, e a CTC a pH 7,0 pela soma dos teores de $\mathrm{Ca}, \mathrm{Mg}, \mathrm{K}$ e $\mathrm{H}+\mathrm{Al}$ (TEDESCO et al., 1995). Os estoques de $\mathrm{CO}$ foram calculados levando-se em consideração a densidade do solo. A análise estatística consistiu na análise da variância segundo o delineamento de blocos ao acaso, sendo a diferença entre médias avaliada pelo teste de Tukey a $5 \%$. A relação entre variáveis foi testada pela significância dos coeficientes de regressões polinomiais.

O solo em SPD apresentou, na camada de 0-6cm, uma concentração de CO 29\% maior do que no SPC, variando esta diferença de $38 \%$, na camada de $0-2$ $\mathrm{cm}$, a $20 \%$, na camada de $4-6 \mathrm{~cm}$ (Figura 1). O aumento do $\mathrm{CO}$, restrito às camadas superficiais do solo, no SPD ocorre devido ao não revolvimento do solo e manutenção dos resíduos vegetais na superfície do solo. Na camada de 0-20cm, o solo em SPD $\left(73,20 \mathrm{t} \mathrm{Cha}^{-1}\right)$ apresentou um estoque de $\mathrm{CO} 2,63 \mathrm{tha}^{-1}$ maior do que o solo sob SPC (70,57 $\left.\mathrm{C} \mathrm{ha}^{-1}\right)$. A taxa de acúmulo de CO em SPD neste solo $\left(0,12 \mathrm{tha}^{-1}\right.$ ano $\left.^{-1}\right)$ pode ser considerada baixa em comparação às taxas de $0,5-0,7 \mathrm{t} \mathrm{ha}^{-1} \mathrm{ano}^{-1}$, determinadas por BAYER et al. (2000), e a taxa de $0,7 \mathrm{t}$ $\mathrm{ha}^{-1}$ ano $^{-1}$, determinada por AMADO et al. (2001), respectivamente, num Argissolo Vermelho $\left(220 \mathrm{~g} \mathrm{~kg}^{-1}\right.$ de argila) e num Argissolo Amarelo (150 $\mathrm{g} \mathrm{kg}^{-1}$ de argila). A baixa taxa de acúmulo de $\mathrm{CO}$ é relacionada à alta estabillidade física da matéria orgânica neste solo

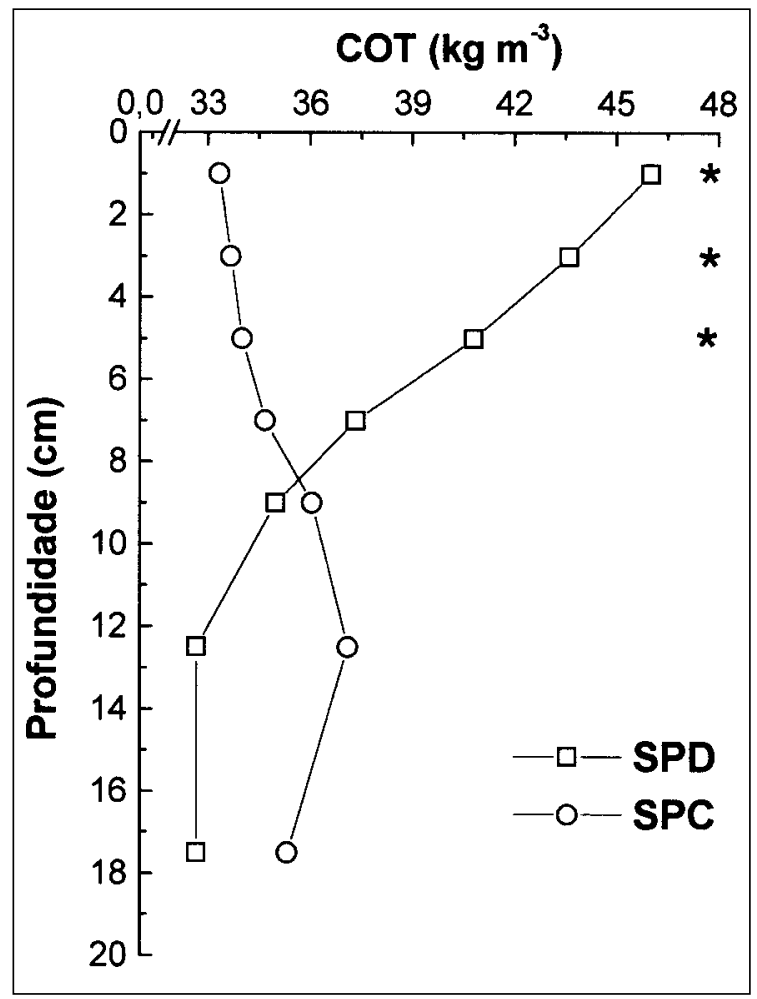

Figura 1 - Distribuição dos teores de carbono orgânico total (CO) no perfil de um Latossolo bruno submetido durante 21 anos aos sistemas de preparo convencional (SPC) e plantio direto (SPD). * indica diferença significativa pelo teste de Tukey a $5 \%$.

argiloso (629 $\mathrm{g} \mathrm{kg}^{-1}$ de argila) e mineralogia oxídica, mesmo quando submetido a intenso revolvimento por lavração e gradagens (SPC).

Apesar de pequeno, o acúmulo de matéria orgânica na superfície do solo em SPD resultou num importante aumento nos valores de CTC efetiva e CTC a pH 7,0 até $8 \mathrm{~cm}$ de profundidade (Tabela 1), em comparação ao SPC. Nas camadas 0-2, 2-4, 4-6 e 6-8cm, o aumento da CTC efetiva variou de $85 \%$ a $17 \%$, e na CTC a pH 7,0 de $31 \%$ a $15 \%$, sendo os maiores incrementos verificados nas camadas superficiais do solo. Na camada de $0-8 \mathrm{~cm}$, ocorreu um aumento médio de $15,2 \mathrm{mmol}_{\mathrm{c}} \mathrm{kg}^{-1}$ na CTC efetiva e de $20,7 \mathrm{mmol}_{\mathrm{c}} \mathrm{kg}^{-1} \mathrm{na}$ $\mathrm{CTC}$ a pH 7,0, o que representou aumentos relativos de $44 \%$ e $21 \%$, respectivamente. A relação entre os teores de $\mathrm{CO}$ e a CTC efetiva e CTC a pH 7,0 do solo pode ser visualizada na figura 2 .

A principal conclusão deste estudo foi que apesar da sua baixa magnitude, o acúmulo de matéria orgânica após 21 anos da adoção do SPD neste solo argiloso e com mineralogia oxídica, promoveu um aumento expressivo na CTC do solo, fundamental neste solo com predominância de minerais de baixa 
Tabela 1 - Valores de CTC efetiva e CTC a pH 7,0 no perfil de um Latossolo bruno submetido durante 21 anos aos sistemas de preparo convencional (SPC) e plantio direto (SPD).

\begin{tabular}{|c|c|c|c|c|}
\hline \multirow{2}{*}{ Profundidade $(\mathrm{cm})$} & \multicolumn{2}{|c|}{ CTC efetiva } & \multicolumn{2}{|c|}{ CTC pH 7,0 } \\
\hline & $\mathrm{SPC}$ & SPD & $\mathrm{SPC}$ & SPD \\
\hline & \multicolumn{4}{|c|}{$\mathrm{mmol}_{\mathrm{c}} \mathrm{kg}^{-1}$} \\
\hline $0-2$ & 34,0 a B & 62,9 a A & 96,7 a B & 126,8 a $\mathrm{A}$ \\
\hline $2-4$ & 35,4 a B & $51,4 \mathrm{~b} \mathrm{~A}$ & 99,3 a B & 123,2 a $\mathrm{A}$ \\
\hline $4-6$ & 34,8 a B & $44,7 \mathrm{bc} \mathrm{A}$ & 98,2 a $\mathrm{A}$ & 112,2 a $\mathrm{A}$ \\
\hline $6-8$ & 35,3 a B & $41,4 \mathrm{~cd} \mathrm{~A}$ & 100,6 a B & 115,4 a $\mathrm{A}$ \\
\hline $8-10$ & 36,2 a $A$ & $38,4 \mathrm{~cd} \mathrm{~A}$ & 98,8 a $\mathrm{A}$ & 107,8 a $\mathrm{A}$ \\
\hline $10-15$ & 35,2 a $\mathrm{A}$ & $38,4 \mathrm{~cd} \mathrm{~A}$ & 99,9 a $\mathrm{A}$ & 110,0 a $\mathrm{A}$ \\
\hline $15-20$ & 34,8 a $\mathrm{A}$ & $36,6 \mathrm{~d} A$ & 96,4 a $\mathrm{A}$ & 103,9 a $\mathrm{A}$ \\
\hline
\end{tabular}

Letras maiúsculas comparam sistemas de preparo dentro da mesma profundidade. Letras minúsculas comparam profundidades dentro de cada sistema de preparo. Médias seguidas de letras iguais não diferem pelo teste de Tukey a 5\%.

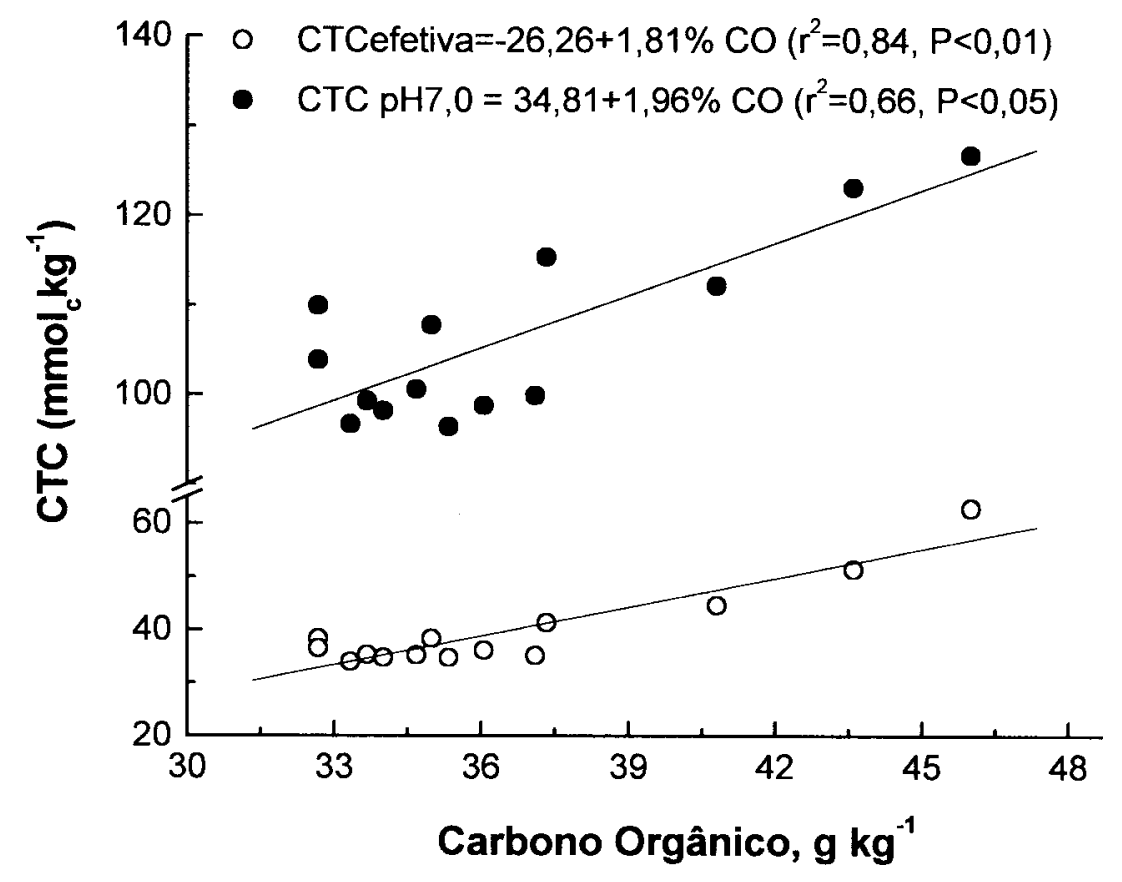

Figura 2 - Relação entre os teores de carbono orgânico (CO) e a CTC efetiva e a pH 7,0 de um Latossolo bruno. Os teores de carbono orgânico correspondem a valores médios (3 repetições) de sete camadas de solo, nos sistemas de plantio direto e preparo convencional.

atividade na fração argila. Além disso, o acúmulo de matéria orgânica restringe-se às camadas superficiais do solo, cuja importância sobre as condições físicas, químicas e biológicas do solo possivelmente não seja refletida pelos baixos valores de acúmulo verificados na camada de $0-20 \mathrm{~cm}$ do solo, na qual o efeito é diluído.

\section{REFERÊNCIAS BIBLIOGRÁFICAS}

AMADO, T.J.C. et al. Potencial de plantas de cobertura em acumular carbono e nitrogênio no solo em sistema de plantio direto e contribuir para a melhoria da qualidade ambiental. R Bras Ci do Solo, v.25, p.128-134, 2001.

Ciência Rural, v. 33, n. 6, nov-dez, 2003. 
BAYER, C. Dinâmica da matéria orgânica em sistemas de manejo de solos. 1996. 241f. Tese (doutorado) Universidade Federal do Rio Grande do Sul.

BAYER, C. et al. Organic matter storage in a sandy clay loam Acrisol affected by tillage and cropping systems in southern Brazil. Soil Till Res, v.54, p.101-109, 2000
EMBRAPA-Centro Nacional de Pesquisa de Solos. Manual de métodos de análise de solo. 2. ed. Rio de Janeiro, 1997. 212p.

TEDESCO, M. J. et al. Análises de solo, plantas e outros materiais. Porto Alegre : Universidade Federal do Rio Grande do Sul, Faculdade de Agronomia 1995. 174p. (Boletim Técnico de Solos, 5). 